\title{
Applying the Analytic Hierarchy Process to an Institutional Repository Collection
}

\author{
Pamela Andrews \\ University of North Texas \\ Denton, Texas \\ pamela.andrews@unt.edu
}

\author{
Karen Harker \\ University of North Texas \\ Denton, Texas \\ karen.harker@unt.edu
}

\author{
Ana Krahmer \\ University of North Texas \\ Denton, Texas \\ ana.krahmer@unt.edu
}

\begin{abstract}
Building the collection of an institutional repository requires a complex understanding of both digital library infrastructure and staff resources, as well as the institution's faculty awareness and attitudes toward self-archiving. For collection development decisions, institutional repository (IR) managers weigh the influence of these factors when pursuing strategies to increase content and faculty participation. To evaluate strategies for collection development, the authors will apply the Analytic Hierarchy Process (AHP) to create a model through which collection development strategies can be evaluated based on the unique context of the institution.
\end{abstract}

\section{CCS CONCEPTS}

- Information systems $\rightarrow$ Digital libraries and archives; • Applied computing $\rightarrow$ Decision analysis;

\section{KEYWORDS}

institutional repository; analytic hierarchy processing; digital libraries

\section{ACM Reference Format:}

Pamela Andrews, Karen Harker, and Ana Krahmer. 2018. Applying the Analytic Hierarchy Process to an Institutional Repository Collection. In FCDL '18: The 18th ACM/IEEE foint Conference on Digital Libraries, fune 3-7, 2018, Fort Worth, TX, USA. ACM, New York, NY, USA, 4 pages. https: //doi.org/10.1145/3197026.3197064

\section{INTRODUCTION}

The institutional repository collection often operates in tandem with an institution's other digital collections, functioning as a portion of the library specifically tailored to preserving and providing access to the scholarly output of an institution. With nearly 400 institutional repositories in the United States alone [8], many digital libraries have developed resources and policies to guide the development of these collections. However, while these collaborations help IR management by providing them numerous strategies to consider and implement, the highly contextual nature of the home institution plays a role in the level of success for these adapted

Permission to make digital or hard copies of all or part of this work for personal or classroom use is granted without fee provided that copies are not made or distributed for profit or commercial advantage and that copies bear this notice and the full citation on the first page. Copyrights for components of this work owned by others than the author(s) must be honored. Abstracting with credit is permitted. To copy otherwise, or republish, to post on servers or to redistribute to lists, requires prior specific permission and/or a fee. Request permissions from permissions@acm.org.

7CDL '18, fune 3-7, 2018, Fort Worth, TX, USA

(C) 2018 Copyright held by the owner/author(s). Publication rights licensed to the Association for Computing Machinery.

ACM ISBN 978-1-4503-5178-2/18/06 . \$15.00

https://doi.org/10.1145/3197026.3197064 strategies. Repository managers must consider variables such as the institution's unique faculty composition, the faculty members' attitudes toward self-archiving policies, institutional policies toward open-access initiatives, the technical infrastructure of the institutional repository, and their own staff resources when deciding upon a collection development strategy specifically for the IR. For the specific context of the IR, collection development is in large part accomplished through mediation practices dealing with the solicitation and collection of materials for the IR. The primary value of the method proposed in this paper lies in its ability to explore the relationship between these variables, making explicit the implied relationships, in a manner that can be understood by the repository's stakeholders, library administrators, and those external to the library.

\subsection{Literature Review}

Analytic Hierarchy Process is a mathematical model for decisionmaking that is based on relative paired-comparisons. Developed in the 1970's by operations researcher Thomas Saaty, it has become a standard tool in engineering and business management. Only recently has it been applied to library and information science, mostly in Asia or Europe. The AHP model applies proportions of ratings of criteria in a square matrix to generate weighted scores of objects being evaluated. For example, Harshan, Chen and Shi assess the quality of library websites to identify attributes that prioritized improvements [4]. Xi, et al. employed AHP to evaluate interlibrary loan service [11]. The weights of the criteria were derived from scores by experts in the field; the measurements used for each of the levels or scores for these criteria were objective and not difficult to assess. For example, for the criterion of Condition of Library there were four attributes, one of which was Collection Quantity, for which there were seven mutually-exclusive and comprehensive levels, for which successively greater scores were applied: $<1,000,000(2) ; 1.0-1.5 M(3) ; 1.51 M-2.0 M(4) \ldots,>3.51 M$. The researchers then applied the aforementioned weights of the criteria to the scores, which were then produced in a cross-matrix, and finally, the sums of the subcriteria matrices were calculated for an overall quality score, Q. Based on the moderately extensive literature review, however, no applications of AHP were found within the library science and information subfields of archival studies or digital libraries.

AHP offers possibilities to institutional repository (IR) managers to solve vexing issues. The 2013 Confederation of Open Access Repositories (COAR) [7] environmental scan of open access repositories identified barriers to depositing works, including faculty awareness, motivation, time, and permissions. The authors of the report encouraged the adoption of sustainable practices to solve 
these: incentivization, repository integration at the institutional services, and assigning a full-time employee to oversee IR operations and assist faculty with deposit [7]. Since this report, the Coalition for Networked Information released a summary of a roundtable session rethinking the nature of institutional repositories, in response to the continual changing landscape of repository services, new platforms arising in competition with as well as complementary to IRs, and the position of IRs within broader open access strategies [3]. Solutions discussed by CNI acknowledged the variety of factors impacting how individual repositories operate. A large portion of the report acknowledges the difficulty of needing firmer strategic goals for the repository before a solution could be found to address how the IR functions within the broader open access and scholarly communication landscape, as well as how faculty needs may also shape what strategic directions are most desired by the institution's community. Local concerns drive a large number of decisions, yet the CNI roundtable reported that despite a large number of librarians present, "only a few institutions discussed assessment of repository programs and no institution present had stated goals or metrics for what constituted success" [3]. This omission points to a need among repository managers for tools to guide assessment and for formulating metrics of success. While both reports describe strategies for repositories, it is striking that the same issues exist four years after the first report, and that similar strategies are recommended in both discussions.

Shifting from the professional organizational level to individual repository managers, Ruth Tillman [10] and Ellen Dubinsky [2] conducted surveys of both repository managers and repositories respectively to examine the many variables associated with repository growth. Both studies imply that despite high-levels of IR manager outreach, faculty participation in the IR is low, and the implications demand further research into faculty buy-in and library retention in IR position. Additionally, the lack of faculty participation despite these efforts can lead to burnout or overall job dissatisfaction with repository management staff due to an increased workload with little results, and a feeling of a lack of control in increasing the effectiveness of IR services [1]. Repository staff can find support and control in their decision-making process by applying a systematic method of evaluating the multiple variables involved in developing the repository collection. The AHP decision-making model can also provide support in justifying a given decision to internal and external stakeholders. By requiring a stated goal as part of the model, repository staff are asked to specify a single goal by which these decisions can be evaluated. Stating the goal is also an essential part of establishing an assessment through by which their later efforts can be evaluated. By making tangible the often implicit factors that impact faculty participation, this model provides a method of gaining clarity regarding a repository staff's effectiveness. Applying AHP to decision-making processes in the evaluation and promotion of institutional repositories could enable repository managers to consider new ways to solicit faculty participation.

\section{RESEARCH DESIGN}

This project examines how the mediation practices for a repository manager's collection development strategy can be evaluated in a systematic way despite being based on multiple variables unique to the individual institution's context by adapting the AHP decisionmaking model for IR managers. This tests the feasibility of adapting AHP to the IR and sets a conceptual foundation for further studies to evaluate the model with a given institution and discuss its implementation. Building the AHP model consists of four processes:

(1) Deciding upon the goal which this decision should meet

(2) Selecting the criteria by which the goal will be measured for success as well as the alternative decisions that will be used to pursue the goal

(3) Weighting these criteria

(4) Comparing scores to determine the suitability of these options in meeting the overall goal.

To visually demonstrate the overall connections of the model, Figure 1 provides an example of the connections between the toplevel goal, the criteria for determining a decision, and the alternative decisions available. The goal, which for the example is to prioritize the effect of mediation practices on the quality of the collection, is then broken down into measures of success as listed in Table 1 and used to score the alternative decisions listed in Table 2 in order to evaluate mediation practices against the individual institutional context so as to best meet the stated goal.

Figure 1: AHP Model Structure

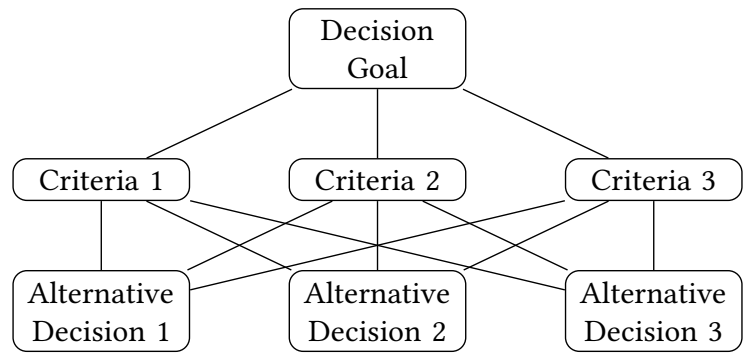

While the model for an institutional repository will have more criteria and alternative decisions than shown, Figure 1 demonstrates how each alternative is considered through each criterion listed rather than against the goal itself. In doing so, each decision is measured against the reality of its operation rather than the perception of its outcome.

For the purpose of building this model, the goal, criteria, and alternative decisions were initially derived from the authors' home institution and experiences with the institutional repository, then discussed and reframed against the broader context of recommendations for repository managers as reviewed previously. However, in adapting this model for other institutions, their own local context will guide the selection of the goal, criteria, and alternative decisions. For example, the metrics available through another institution's IR may limit or expand possible criteria for inclusion. While adapting the model will require strategic planning on the part of repository management staff at another institution, this model proves an example of what it would look like to employ this systematic process for evaluating decisions for IR collection development. Adapting the AHP model structure to fit the decisions and criteria considered by repository managers also pushes the translation of anecdotal data and qualitative landmarks of success into concrete, 
codified elements to be considered against each other. The goal of this project is to demystify AHP as a method for library use, and to provide a working example to ease the labor in translating IR strategies.

\subsection{Criteria and Decision Selection}

From the repository management perspective mediation practices are both informed by staff understanding of faculty attitudes and behavior, as well as by the technical infrastructure of the repository. As the example institution does not have a mechanism for faculty to self-submit items to the repository, instead sending items to the repository manager for preparation and uploading, mediation practices are primarily considered with processes for gathering materials for inclusion in the IR. The top level of the hierarchy is the decision goal for the repository. As stated previously, for this example the goal is to prioritize the effect of mediation practices on the quality of the collection. As reviewed previously, repository managers have a number of organizations and studies to consult for strategies to increase the repository collection. This process will allow for prioritizing strategies to understand how one might better fit the institution's context over another.

For the purpose of this example, quality was decided by the authors to consist of three criteria as seen in Table 1: dissemination of knowledge, impact on reputation, and efficiency. These criteria relate to the IR as a part of open access initiatives to increase access to knowledge, speak to the IR as a way to showcase the research output of a given institution, and include a pragmatic understanding of the technical infrastructure and human resources available for maintaining the IR, elements outlined in both the COAR and CNI reports.

Table 1: AHP criteria as measures of success for quality

\begin{tabular}{ll}
\hline AHP Level & Measure of Success \\
\hline Criterion 1 & Dissemination of knowledge \\
Sub-criterion 1.1 & Rarity of items \\
Sub-criterion 1.2 & Distribution of subjects \\
Sub-criterion 1.3 & Direct uses \\
Criterion 2 & Impact on reputation \\
Sub-criterion 2.1 & Indirect use \\
Sub-criterion 2.2 & Grant-funded research prod- \\
& ucts \\
Criterion 3 & Efficiency \\
Sub-criterion 3.1 & Low cost per unit input \\
\hline
\end{tabular}

As these criteria are broad, sub-criteria have been included to further define how these criteria will be met. The dissemination of knowledge can further be understood by the rarity of items collected (whether they are only available in the repository or elsewhere, as well as risk of being lost due to technological obsolescence), whether this knowledge is highly specialized or has representation from the variety of subjects and disciplines studied by the institution, and direct uses of these items to understand their discoverability and usage. Impact on reputation focuses on the IR as a way to facilitate the application of collected items to further research, and was determined to be measured by indirect usage (such as by citations and links to items in other scholarship) as well as its relationship with facilitating funded research projects. The criterion of efficiency applies to whether a decision leads to the greatest outcomes for the least effort, particularly with the time and labor cost for item gathered.

Many repository managers and professional organizations recommend a combination of different tactics. However, including specific tactics (e.g. systematic review of faculty CVs for items to upload) as alternative decisions within the model places them in competition with each other. Specific tactics also require more specific criteria for evaluation that would crowd the model, making it less useful for broadly evaluating decisions against the overall goal. Instead, these tactics have been arranged around a leading emphasis to evaluate whether tactics in these groups would be more or less useful for meeting the goal. After evaluating the model and choosing the alternative decision, the repository manager then has a very targeted lens through which they can select tactics rather than being overwhelmed by the many recommendations. The areas of emphasis have been listed in Table 2 as alternative decisions.

Table 2: AHP alternative decisions for implementing mediation practices

\begin{tabular}{ll}
\hline AHP Level & Mediation Practice \\
\hline Alternative Decision 1 & System focused processes \\
Alternative Decision 2 & Partner focused processes \\
Alternative Decision 3 & Champion focused processes \\
\hline
\end{tabular}

Tactics that focus on leveraging the technical infrastructure or primarily shift the labor of gathering items onto the repository manager (such as a systematic review of all faculty CVs rather than relying on faculty to approach the repository manager) fall under system-focused processes. Strategies that involve leveraging institutional partners, such as items gathered by the Graduate School for electronic theses and dissertations or the Honors College for senior theses or honors capstone works, would fall under partner-focused processes. Champion-focused processes rely on recruiting selected individuals across campus and using this network to promote and gather items for the IR.

Stating each criterion's relationship to the goal also helps stakeholders understand how large a role it plays in measuring the value of the decision in relation to the goal. If the weights assigned to the criteria need to be adjusted during the consistency analysis, stakeholders will need to understand the relationship of the criteria to the goal and possibly consider whether the goal itself should be modified.

\subsection{Implementation}

After constructing the hierarchy, repository staff can weight the criteria to codify the relationships between hierarchy levels in the decision-making process. Following the approach of Nakatani and Chuang [6], AHP is implemented through the following steps:

(1) Create a pairwise comparison matrix with a scale of 1-9 to compare the criteria and/or subcriteria and assign a value demonstrating their importance in relation to the others in terms of meeting the goal. 
(2) After completing all comparisons for the model, normalize the scores to obtain the eigenvector of the matrix.

(3) Repeat this process for the next level down, with an additional pair-wise comparison matrix comparing each alternative with respect to the sub-criterion at the next higher level.

(4) Once all comparisons have been weighted and calculated, synthesize these matrices to understand the overall priorities.

AHP itself is not a complicated procedure. The bulk of the work for repository staff is translating the context and resources of their own institution into hierarchical levels and then weighting the criteria as appropriate for their institution.

As this model contains a large degree of subjective judgment, some comparisons may seem inconsistent. Whereas one criteria, such the low cost per unit input, may be deemed more important than the rarity of the content collected, it may be less important than direct usage, but not necessarily by the same amount. In their summary of AHP, Mu and Pereyra-Rojas [5] recommend that users calculate consistency, using a consistency ratio in which the consistency index of the created matrix is compared to the consistency index of a random-like matrix. Saaty [9] has provided values for the random-like matrix, which is the average consistency index of 500 randomly filled matrices. A consistency ratio of 0.10 or less indicates a reasonably consistent matrix. Scores higher than 0.10 should be re-evaluated to locate areas of increased inconsistency [5].

$\mathrm{Mu}$ and Pereyra-Rojas [5] also recommend a sensitivity analysis to give further context to decisions made using AHP. For this analysis, another pair-wise comparison matrix is created, but with different weighted values to represent a what-if scenario. For example, all weighted values may be made the same to consider how the alternatives rank with all factors considered equal. This can be used to demonstrate to administration how the nature of an individual institution's context can influence values, particularly if the decision recommended with all factors weighted equally was determined to be unsuccessful in the past.

\section{APPLICATIONS}

The flexible nature of the criteria and alternative decisions allow this model to be applied in a number of different scenarios for repository management staff. While this project is concerned with collection development through mediation practices, it is not limited to these types of decisions. As more repository managers learn and share new methods for increasing collection engagement, the authors believe this will be a useful tool for testing the applicability of these methods at other institutions before expending the time and resources on implementation. As outlined here, this model can be used as constructed and recalculated according to another institution's priorities. However, it is also flexible enough to be repopulated with another institution's own criteria for their goal. For example, it could be re-purposed to evaluate marketing strategies suggested for increasing faculty awareness of the IR. After a decision has been implemented, its relative success or failure can also be used to adjust the AHP model. Ultimately, this model is recommended to aid repository staff in justifying their decisions through a systematic evaluation of the decisions available, and the strengths and weaknesses of the institution's existing resources.

\section{CONCLUSIONS}

Institutional repository managers require a complex understanding of both their own resources and the larger campus context to best to build the collection. The highly contextual nature of their work means that practices shared among managers are not likely to have consistent results at multiple institutions. Applying AHP to the multifaceted nature of repository collection building attempts to provide a standard procedure for how managers can make decisions for collection-planning and building based upon their context, rather than upon anticipated success based on a different institution's outcomes. AHP enables managers to balance, through weighted criteria, degrees of contexts and the goal of their respective institutions toward planning around needs and resources. The AHP approach, with weighted criteria, helps IR managers rank relevance of current strengths and desired goals toward repository planning and decision-making.

\section{REFERENCES}

[1] L. Christian. 2015. A Passion Deficit: Occupational Burnout and the New Librarian: A Recommendation Report. The Southeastern Librarian 62, 4 (2015), 2-11. https: //digitalcommons.kennesaw.edu/seln/vol62/iss4/2/

[2] Ellen Dubinsky. 2014. A Current Snapshot of Institutional Repositories: Growth Rate, Disciplinary Content and Faculty Contributions. Fournal of Librarianship and Scholarly Communication 2, 3 (2014). http://dx.doi.org/10.7710/2162-3309. 1167

[3] Coalition for Networked Information. 2017. Rethinking Institutional Repository Strategies. (2017). https://www.cni.org/wp-content/uploads/2017/05/ CNI-rethinking-irs-exec-rndtbl.report.S17.v1.pdf

[4] Ramanayaka Kokila Harshan, Xianqiao Chen, and Bing Shi. 2016. Analytic Hierarchy Process (AHP) based model for assessing performance quality of library websites. Information Technology fournal 16, 1 (December 2016), 35-43. https://doi.org/10.3923/itj.2017.35.43

[5] Enrique $\mathrm{Mu}$ and Milagros Pereyra-Rojas. 2017. Practical Decision Making. SpringerBriefs in Operation Research, Vol. 2. Springer International Publishing, New York, Chapter Understanding the Analytic Hierarchy Process, 7-22. https://doi.org/10.1007/978-3-319-33861-3_2

[6] Kazuo Nakatani and Ta-Tao Chuang. 2011. A web analytics tool selection method: an analytical hierarchy process approach. Internet Research 21, 2 (January 2011), 171-186. https://doi.org/10.1108/10662241111123757

[7] Confederation of Open Access Repositories. 2013. Incentives, Integration, and Mediation: Sustainable practices for populating repositories report. (2013). https://www.coar-repositories.org/news-media/publications/ sustainable-practices-for-populating-repositories-report/

[8] Directory of Open Access Repositories. 2018. List of Repositories - United States. (2018). http://www.opendoar.org/countrylist.php?cContinent=North\% 20America\#United $\% 20$ States

[9] Thomas L. Saaty. 2012. Decision Making for Leaders: The Analytic Hierarchy Process for Decisions in a Complex World (third revised ed.). RWS Publications, Pittsburgh, PA.

[10] R. K. Tillman. 2017. Where Are We Now? Survey on Rates of Faculty SelfDeposit in Institutional Repositories. Fournal of Librarianship and Scholarly Communication 5, 1 (December 2017), 1-27. https://doi.org/10.7710/2162-3309. 2203

[11] Qingkui Xi, Qian Zhang, Feng Ni, Guiting Cha, and Ping Bao. 2015. Promoting ILL/DD within the Jiangsu Academic Library \& Information System in China by small consortia. Interlending \& Document Supply 43, 4 (August 2015), 182-188. https://doi.org/10.1108/ILDS-08-2015-0024 